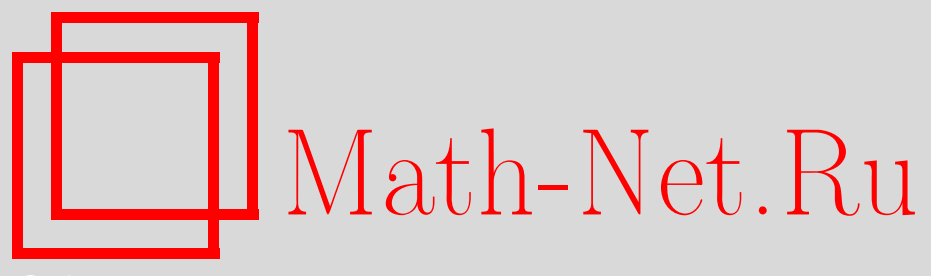

В. Б. Диденко, О спектральных свойствах дифференциальных операторов с неограниченными операторными коэффициентами, определяемых линейным отношением, Матем. заметки, 2011, том 89, выпуск 2, 226-240

DOI: https://doi.org/10.4213/mzm6612

Использование Общероссийского математического портала Math-Net.Ru подразумевает, что вы прочитали и согласны с пользовательским соглашением http://www . mathnet.ru/rus/agreement

Параметры загрузки:

IP : 34.229 .45 .116

26 апреля 2023 г., 13:13:51

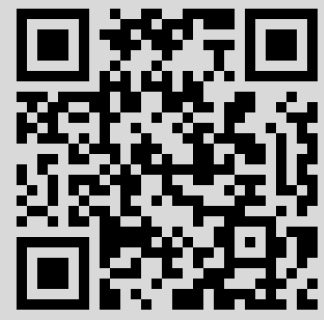


Том 89 выпуск 2 февраль 2011

УДК 517

\section{О спектральных свойствах дифференциальных операторов с неограниченными операторными коэффициентами, определяемых линейным отношением}

\section{В. Б. Диденко}

Получены необходимые и достаточные условия обратимости и фредгольмовости операторов, порожденных семейством эволюционных операторов и краевыми условиями, заданными с помощью линейного отношения.

Библиография: 7 названий.

1. Введение. Пусть $X$ - банахово пространство над полем $\mathbb{K} \in\{\mathbb{R}, \mathbb{C}\}$ и End $X-$ банахова алгебра эндоморфизмов пространства $X$.

Символом $\Delta$ обозначим множество $[a, b] \times[a, b]$. Отображение $\mathscr{U}: \Delta \rightarrow$ End $X$ называется (сильно непрерывным) семейством эволюиионных операторов на $[a, b]$, если выполнены следующие условия:

(1) $\mathscr{U}(t, t)=I$ - тождественный оператор для любого $t \in[a, b]$;

(2) $\mathscr{U}(t, s) \mathscr{U}(s, \tau)=\mathscr{U}(t, \tau)$ для всех $t, s, \tau$ из $[a, b]$;

(3) отображение $(t, s) \mapsto \mathscr{U}(t, s) x: \Delta \rightarrow X$ непрерывно для любого $x \in X$.

Символом $L_{p}=L_{p}([a, b], X), 1 \leqslant p \leqslant \infty$, будем обозначать (банахово) пространство классов измеримых по Бохнеру функций, для которых конечна величина (принимаемая за норму в соответствующем пространстве)

$$
\|x\|_{p}=\left(\int_{a}^{b}\|x(\tau)\|^{p} d \tau\right)^{1 / p}, \quad p \neq \infty, \quad\|x\|_{\infty}=\operatorname{ess} \sup _{\tau \in[a, b]}\|x(\tau)\| .
$$

Через $C=C([a, b], X)$ обозначим (банахово) пространство непрерывных на $[a, b]$ функций, принимающих значения в $X$.

Символом $\mathscr{F}=\mathscr{F}([a, b], X)$ будем обозначать одно из перечисленных выше пространств.

Пусть $\Gamma$ - произвольное линейное отношение на $X$, т.е. $\Gamma$ - некоторое линейное подпространство из $X \times X$. В данной работе рассматривается оператор

$$
\mathscr{L}_{\Gamma}: D\left(\mathscr{L}_{\Gamma}\right) \subset \mathscr{F} \rightarrow \mathscr{F},
$$

который определяется следующим образом. Непрерывная функция $x:[a, b] \rightarrow X$, для которой $(x(a), x(b)) \in \Gamma$, включается в $D\left(\mathscr{L}_{\Gamma}\right)$, если существует функция $f \in \mathscr{F}$

Работа выполнена при поддержке Российского фонда фундаментальных исследований (грант № 10-01-00276).

(C) В. Б. Диденко, 2011 
такая, что верны равенства

$$
x(t)=\mathscr{U}(t, a) x(a)+\int_{a}^{t} \mathscr{U}(t, s) f(s) d s, \quad t \in[a, b] .
$$

При этом полагается $\mathscr{L}_{\Gamma} x=f$. Отметим корректность определения оператора $\mathscr{L}_{\Gamma}$ (т.е. единственность функции $f$, построенной по $x$ ).

Одним из примеров операторов, задаваемых с помощью равенств (1.1), является дифференциальный оператор $\mathscr{L}=d / d t-A$ в случае, когда оператор $A$ является генератором сильно непрерывной группы операторов $T: \mathbb{R} \rightarrow \operatorname{End} X$. В этом случае семейство эволюционных операторов имеет вид $\mathscr{U}(t, s)=T(t-s)$.

Часто граничные условия задаются с помощью упорядоченной пары линейных операторов $(A, B)$ (см. [1]), где операторы $A, B$ из $\operatorname{End} X$, когда для линейного отношения $\Gamma$ справедливо одно из следующих представлений:

$$
\begin{aligned}
& \Gamma=\{(A x, B x), x \in X\}), \\
& \Gamma=\{(x, y) \in X \times X: A x=B y\} .
\end{aligned}
$$

Если отношение Г является несингулярным (определение будет дано ниже), то оно может быть представлено как в виде (1.2), так и в виде (1.3) (см. теорему 5). В случае, когда $X$ - конечномерное пространство, любое линейное отношение $\Gamma \subset$ $X \times X$ представимо в виде (1.2), либо в виде (1.3). Это утверждение следует из теорем 6 и 7.

Используемые в следующем определении понятия будут введены ниже.

ОПреДЕЛЕНИЕ 1. Пусть $\mathscr{A}$ из $\operatorname{LR}(\mathscr{X})$, где $\mathscr{X}$ - банахово пространство и для отношения $\mathscr{A}$ выполнено хотя бы одно из следующих условий:

1) $\mathscr{A} \in \operatorname{LRC}(\mathscr{X})$;

2) $\operatorname{Ker} \mathscr{A}=\{0\}$, т.е. отношение $\mathscr{A}$ инъективно;

3) отношение $\mathscr{A}$ корректно;

4) $\alpha(\mathscr{A})=\operatorname{dim} \operatorname{Ker} \mathscr{A}<\infty$;

5) $\operatorname{Ker} \mathscr{A}$ - замкнутое дополняемое подпространство в $\mathscr{X}$;

6) $\overline{\operatorname{Im} \mathscr{A}}=\operatorname{Im} \mathscr{A}$;

7) $\operatorname{Im} \mathscr{A}$ - замкнутое дополняемое в $\mathscr{X}$ подпространство;

8) $\operatorname{Im} \mathscr{A}$ - замкнутое подпространство конечной коразмерности $\operatorname{Codim} \operatorname{Im} \mathscr{A}=$ $\beta(\mathscr{A})$;

9) $\operatorname{Im} \mathscr{A}=\mathscr{X}$, т.е. $\mathscr{A}$ - сюръективное отношение;

10) отношение $\mathscr{A}$ непрерывно обратимо.

Если для $\mathscr{A}$ выполнены все условия из совокупности условий $S=\left\{i_{1}, \ldots, i_{k}\right\}$, где $1 \leqslant i_{1}<i_{2}<\cdots<i_{k} \leqslant 10$, то будем говорить, что отношение $\mathscr{A}$ находится в состоянии обратимости $S$.

В частности, если отношение $\mathscr{A} \in \operatorname{LR}(\mathscr{X})$ находится в состоянии обратимости $F=\{4,8\}$, то отношение $\mathscr{A}$ будет фредгольмовым. Индексом фредгольмова отношения $\mathscr{A}$ назовем число $\operatorname{ind}(\mathscr{A})=\alpha(\mathscr{A})-\beta(\mathscr{A})$.

В работе получены следующие результаты.

Теорема 1. Если для оператора $\mathscr{L}_{\Gamma}$ выполнено одно из приведенных в определении 1 десяти свойств, то соответствующим свойством обладает отношение $\Gamma-\mathscr{U}(b, a)$. Любое из десяти свойств определения 1 , выполненное для отношения 
$\Gamma-\mathscr{U}(b, a)$, исключая, быть может, первое и пятое свойства, выполнено и для onepamopa $\mathscr{L}_{\Gamma}$.

Tеорема 2. Onератор $\mathscr{L}_{\Gamma} \oint$ федголъмов тогда и толъко тогда, когда фредгольмовым является отношение $Г-\mathscr{U}(b, a)$. Если один из них фредгольмов, то

$$
\alpha\left(\mathscr{L}_{\Gamma}\right)=\alpha(\Gamma-\mathscr{U}(b, a)), \quad \beta\left(\mathscr{L}_{\Gamma}\right)=\beta(\Gamma-\mathscr{U}(b, a)),
$$

а значит, и их индексы совпадают.

2. Некоторые сведения из теории линейных отношений. Пусть $X$ - банахово пространство над полем $\mathbb{K} \in\{\mathbb{R}, \mathbb{C}\}$. Любое линейное подпространство $\mathscr{A} \subseteq X \times X$ называется линейным отношением на пространстве $X$. Множество всех замкнутых линейных отношений на $X$ будем обозначать $\operatorname{LRC}(X)$. Множество всех линейных отношений на $X$ будем обозначать $\operatorname{LR}(X)$. Линейные операторы будут часто отождествляться с их графиком, т.е. рассматриваться в качестве линейных отношений. Используемые далее понятия из теории линейных отношений (многозначных линейных операторов) можно найти в монографии [2] и в статье [3].

Областью определения $D(\mathscr{A})$ отношения $\mathscr{A} \in \mathrm{LR}(X)$ называется подпространство вида $D(\mathscr{A})=\{x \in X:(x, y) \in \mathscr{A}$ для некоторого $y \in X\}$.

Образом $\operatorname{Im} \mathscr{A}$ отношения $\mathscr{A} \in \operatorname{LR}(X)$ называется подпространство вида $\operatorname{Im} \mathscr{A}=$ $\{y \in X:(x, y) \in \mathscr{A}$ для некоторого $x \in X\}$.

Ядром линейного отношения $\mathscr{A}$ из $\operatorname{LR}(X)$ называется множество $\operatorname{Ker} \mathscr{A}=\{x \in$ $D(\mathscr{A}):(x, 0) \in \mathscr{A}\}$.

Для отношения $\mathscr{A} \in \mathrm{LR}(X)$ и элемента $x \in X$ определим множество вида $\mathscr{A} x=$ $\{y:(x, y) \in \mathscr{A}\}$. В частности, множество $\mathscr{A} 0$ имеет вид $\mathscr{A} 0=\{y:(0, y) \in \mathscr{A}\}$.

Суммой двух линейных отношений $\mathscr{A}_{1}, \mathscr{A}_{2}$ из $\operatorname{LR}(X)$ называется линейное отношение вида

$$
\mathscr{A}_{1}+\mathscr{A}_{2}=\left\{(x, y) \in X \times X: x \in D\left(\mathscr{A}_{1}\right) \cap D\left(\mathscr{A}_{2}\right), y \in \mathscr{A}_{1} x+\mathscr{A}_{1} x\right\} .
$$

Здесь под $\mathscr{A}_{1} x+\mathscr{A}_{2} x$ понимается алгебраическая сумма двух множеств $\mathscr{A}_{1} x, \mathscr{A}_{2} x$. Из этого определения следует, что $D\left(\mathscr{A}_{1}+\mathscr{A}_{2}\right)=D\left(\mathscr{A}_{1}\right) \cap D\left(\mathscr{A}_{2}\right)$.

Обратным к линейному отношению $\mathscr{A} \in \mathrm{LR}(X)$ называется линейное отношение $\mathscr{A}^{-1}=\{(y, x) \in X \times X:(x, y) \in \mathscr{A}\}$.

Отношение $\mathscr{A}$ из $\operatorname{LRC}(X)$ называется непрерьвно обратимым, если $\mathscr{A}^{-1} \in$ End $X$, т.е. $\operatorname{Ker} \mathscr{A}=\{0\}$ (отношение $\mathscr{A}$ инъективно) и $\operatorname{Im} \mathscr{A}=X$ (отношение $\mathscr{A}$ сюрьективно).

Линейное отношение $\mathscr{A}$ из $\operatorname{LR}(X)$ называется фрредольмовым, если его ядро $\operatorname{Ker} \mathscr{A}$ конечномерно, образ $\operatorname{Im} \mathscr{A}$ замкнут и его коразмерность $\beta(\mathscr{A})=\operatorname{dim}(X / \operatorname{Im} \mathscr{A})$ конечна. Число ind $\mathscr{A}=\alpha(\mathscr{A})-\beta(\mathscr{A})$, где $\alpha(\mathscr{A})=\operatorname{dim} \operatorname{Ker} \mathscr{A}$, называется индексом фредгольмова отношения $\mathscr{A}$.

Нормой линейного отношения $\mathscr{A}$ из $\operatorname{LR}(X)$ назовем число

$$
\|\mathscr{A}\|=\sup _{\|x\| \leqslant 1}\|\mathscr{A} x\|, \quad \text { где } \quad\|\mathscr{A} x\|=\inf _{y \in \mathscr{A} x}\|y\| .
$$

В случае конечности этой величины отношение $\mathscr{A}$ называется ограниченным.

Для отношения $\mathscr{A}$ из $\operatorname{LR}(X)$ величина

$$
\gamma(\mathscr{A})=\sup _{\lambda \in \mathbb{R}_{+}}\{\|\mathscr{A} x\| \geqslant \lambda d(x, \operatorname{Ker} \mathscr{A}) \text { для всех } x \in D(\mathscr{A})\},
$$

где $d(x, \operatorname{Ker} \mathscr{A})=\inf _{x_{0} \in \operatorname{Ker} \mathscr{A}}\left\|x-x_{0}\right\|$, называется инфимумом модуля отношения $\mathscr{A}$. 
В [2] была доказана следующая теорема.

Теорема 3. Для любого отношения $\mathscr{A}$ из $\operatorname{LR}(X)$ справедлива формула

$$
\gamma(\mathscr{A})=\frac{1}{\left\|\mathscr{A}^{-1}\right\|}
$$

Отношение $\mathscr{A}$ из $\operatorname{LR}(X)$ называется корректным (равномерно инбективным), если $\operatorname{Ker} \mathscr{A}=\{0\}$ и $\gamma(\mathscr{A})>0$.

Резольвентным множеством отношения $\mathscr{A} \in \mathrm{LRC}(X)$ называется множество $\rho(\mathscr{A})$ всех $\lambda \in \mathbb{K}$, для которых $(\mathscr{A}-\lambda I)^{-1} \in \operatorname{End} X$. Спектром отношения $\mathscr{A} \in$ $\operatorname{LRC}(X)$ называется множество $\sigma(\mathscr{A})=\mathbb{K} \backslash \rho(\mathscr{A})$.

Пусть операторы $F, G$ из End $X$. K резольвентному множеству $\rho(G, F)$ упорядоченной пары операторов $(G, F)$ отнесем все числа $\lambda$ из $\mathbb{K}$, для которых оператор $G-\lambda F$ непрерывно обратим. Множество $\sigma(G, F)=\mathbb{K} \backslash \rho(G, F)$ назовем спектром этой nарьь.

Для упорядоченной пары операторов $(G, F)$ введем отношения $\mathscr{A}_{l}=F^{-1} G$ и $\mathscr{A}_{r}=G F^{-1}$, которые назовем соответственно левым и правым отношениями для упорядоченной пары $(G, F)$.

В [4] была доказана следующая теорема.

ТЕОрема 4. Если для упорядоченной пары операторов $(G, F)$ множество $\rho(G, F)$ непусто, то справедливы следующие равенства:

$$
\sigma(G, F)=\sigma\left(\mathscr{A}_{l}\right)=\sigma\left(\mathscr{A}_{r}\right)
$$

Отметим, что для упорядоченной пары операторов $(G, F)$ выполняются следующие равенства:

$$
\begin{aligned}
\left(\mathscr{A}_{l}-\lambda I\right)^{-1} & =(G-\lambda F)^{-1} F, & & \lambda \in \mathbb{K}, \\
\left(\mathscr{A}_{r}-\lambda I\right)^{-1} & =F(G-\lambda F)^{-1}, & & \lambda \in \mathbb{K} .
\end{aligned}
$$

Отношение $\mathscr{A}$ из $\operatorname{LRC}(X)$ называется несингулярным, если его резольвентное множество $\rho(\mathscr{A})$ непусто. В противном случае отношение $\mathscr{A}$ называется сингулярHols.

Tеорема 5. Если отношение $\mathscr{A}$ из $\operatorname{LRC}(X)$ является несингулярным, то оно представимо как в виде (1.2), так и в виде (1.3).

ДокАЗАТЕЛЬСтво. Пусть $\lambda_{0} \in \rho(\mathscr{A})$. Тогда для него справедливы следующие представления:

$$
\begin{aligned}
& \mathscr{A}=\{(A x, B x), x \in X\})=B A^{-1}, \\
& \mathscr{A}=\{(x, y) \in X \times X: C x=D y\}=D^{-1} C,
\end{aligned}
$$

где

$$
\begin{aligned}
& A=\left(\mathscr{A}-\lambda_{0} I\right)^{-1}, \quad B=I+\lambda_{0}\left(\mathscr{A}-\lambda_{0} I\right)^{-1}, \\
& C=I+\lambda_{0}\left(\mathscr{A}-\lambda_{0} I\right)^{-1}, \quad D=\left(\mathscr{A}-\lambda_{0} I\right)^{-1} .
\end{aligned}
$$

Представления проверяются непосредственной подстановкой. Теорема доказана.

Пусть теперь $X$ - конечномерное пространство. В [5] были доказаны следующие две теоремы. 
Теорема 6. Для того, чтоби произволъное отношение $\mathscr{A} \in \operatorname{LR}(X)$ было представимо в виде (1.2) для некоторых операторов $A, B \in \operatorname{End} X$, необходимо и достаточно, чтобы выполнялось условие

$$
\operatorname{dim} D(\mathscr{A})+\operatorname{dim} \mathscr{A} 0 \leqslant \operatorname{dim} X .
$$

Теорема 7. Для того, чтоби произвольное отношение $\mathscr{A} \in \operatorname{LR}(X)$ было представимо в виде (1.3) для некоторых операторов $A, B \in \operatorname{End} X$, необходимо и достаточно, чтобы выполнялось условие

$$
\operatorname{dim} D(\mathscr{A})+\operatorname{dim} \mathscr{A} 0 \geqslant \operatorname{dim} X .
$$

3. Оператор $\mathscr{L}_{\Gamma}$ и отношение $\Gamma-\mathscr{U}(b, a)$. Утверждения этого пункта связаны с доказательством утверждений о том, что большинство свойств из определения 1 выполняются для оператора $\mathscr{L}_{\Gamma}$ и отношения $\Gamma-\mathscr{U}(b, a)$ одновременно.

Введем в рассмотрение следующие два оператора: $\mathscr{B}: X \rightarrow \mathscr{F}, \mathscr{C}: \mathscr{F} \rightarrow X$ с помощью равенств

$$
\begin{gathered}
(\mathscr{B} x)(s)=\frac{1}{b-a} \mathscr{U}(s, b) x, \quad s \in[a, b], \quad x \in X, \\
\mathscr{C} f=\int_{a}^{b} \mathscr{U}(b, s) f(s) d s, \quad f \in \mathscr{F} .
\end{gathered}
$$

Лемма 1. Операторы $\mathscr{B}$ и $\mathscr{C}$ ограниченъ.

ДокАЗАТЕльСТво. По определению эволюционного семейства $\mathscr{U}$ для любого элемента $x$ из $X$ функция $(t, s) \mapsto U(t, s) x: \Delta \rightarrow X$ является непрерывной, а значит, она ограничена на $\Delta$. В силу принципа равномерной ограниченности (см. [6; теорема II.2.11]) следует, что существует такое число $M$, для которого $\|\mathscr{U}(t, s)\| \leqslant M$ для любой пары $(t, s)$ из $\Delta$. Отсюда следует ограниченность операторов $\mathscr{B}$ и $\mathscr{C}$.

Непосредственно из определения операторов $\mathscr{B}$ и $\mathscr{C}$ вытекает следующая

Лемма 2. Произведение операторов $\mathscr{C} \mathscr{B}$ является тождественным оператором в $X$.

Лемма 3. Для оператора $\mathscr{L}_{\Gamma}$ справедливы равенства

$$
\begin{aligned}
\operatorname{Ker} \mathscr{L}_{\Gamma} & =\left\{x \in \mathscr{F}: x(t)=\mathscr{U}(t, a) x_{0}, x_{0} \in \operatorname{Ker}(\Gamma-\mathscr{U}(b, a)), t \in[a, b]\right\}, \\
\operatorname{Im} \mathscr{L}_{\Gamma} & =\{f \in \mathscr{F}: \mathscr{C} f \in \operatorname{Im}(\Gamma-\mathscr{U}(b, a))\} .
\end{aligned}
$$

ДокАзАтеЛьство. Докажем справедливость равенства (3.3). Возьмем произвольную функцию $x$ из $\operatorname{Ker} \mathscr{L}_{\Gamma}$. Тогда по определению оператора $\mathscr{L}_{\Gamma}$ справедливо равенство $x(t)=\mathscr{U}(t, a) x_{0}, t \in[a, b]$. Так как $(x(a), x(b)) \in \Gamma$, то получим, что $\left(x_{0}, \mathscr{U}(b, a) x_{0}\right) \in \Gamma$, а значит, $x_{0} \in \operatorname{Ker}(\Gamma-\mathscr{U}(b, a))$.

Непосредственно из определения оператора $\mathscr{L}_{\Gamma}$ следует, что функция $x$, заданная равенством $x(t)=\mathscr{U}(t, a) x_{0}, t \in[a, b]$, где $x_{0}$ из $\operatorname{Ker}(\Gamma-\mathscr{U}(b, a))$, принадлежит Ker $\mathscr{L}_{\Gamma}$. Таким образом, мы доказали равенство (3.3).

Докажем справедливость равенства (3.4). Пусть функция $f$ из $\operatorname{Im} \mathscr{L}_{\Gamma}$. Тогда найдется непрерывная функция $x$, для которой выполняется равенство (1.1) и, кроме того, $(x(a), x(b))$ принадлежит Г. Отсюда следует, что $(x(a), \mathscr{U}(b, a) x(a)+\mathscr{C} f) \in \Gamma$, а значит, $\mathscr{C} f \in \operatorname{Im}(\Gamma-\mathscr{U}(b, a))$. 
Пусть теперь $\mathscr{C} f \in \operatorname{Im}(\Gamma-\mathscr{U}(b, a))$. Рассмотрим функцию $x$, определяемую равенством

$$
x(t)=\mathscr{U}(t, a) x_{0}+\int_{a}^{t} \mathscr{U}(t, s) f(s) d s, \quad t \in[a, b],
$$

где $x_{0}$ из $(\Gamma-\mathscr{U}(b, a))^{-1}(\mathscr{C} f)$. Для построенной таким образом функции $x$ справедливо равенство (1.1), и кроме того, пара $(x(a), x(b))$ принадлежит $\Gamma$, а значит, $\mathscr{L}_{\Gamma} x=f$. Следовательно $f \in \operatorname{Im} \mathscr{L}_{\Gamma}$. Таким образом, мы доказали равенство (3.4). Лемма доказана.

Лемма 4. Образ $\operatorname{Im} \mathscr{L}_{\Gamma}$ оператора $\mathscr{L}_{\Gamma}$ замкнут тогда и толъко тогда, когда замкнут образ $\operatorname{Im}(\Gamma-\mathscr{U}(b, a))$ отношения $\Gamma-\mathscr{U}(b, a)$.

ДокАзАтельство. Пусть замкнут образ оператора $\mathscr{L}_{\Gamma}$. Покажем замкнутость образа отношения $\Gamma-\mathscr{U}(b, a)$. Пусть $\left(x_{n}\right)$ - сходящаяся к $x_{0}$ последовательность из $\operatorname{Im}(\Gamma-\mathscr{U}(b, a))$. Рассмотрим последовательность функций $\left(f_{n}\right)$ из $\mathscr{F}$, определенных по правилу $f_{n}=\mathscr{B} x_{n}, n \geqslant 1$.

Поскольку элемент $\mathscr{C} f_{n}=x_{n}$ из $\operatorname{Im}(\Gamma-\mathscr{U}(b, a))$ для любого натурального $n$, то $f_{n} \in \operatorname{Im} \mathscr{L}_{\Gamma}$. Так как в силу ограниченности оператора $\mathscr{B}$ последовательность $\left(f_{n}\right)$ сходится к функции $f_{0}=\mathscr{B} x_{0}$, то в силу леммы 3 , замкнутости образа оператора $\mathscr{L}_{\Gamma}$ и равенства $\mathscr{C} f_{0}=x_{0}$ получаем, что $x_{0}$ принадлежит $\operatorname{Im}(\Gamma-\mathscr{U}(b, a))$.

Пусть замкнут образ отношения $\Gamma-\mathscr{U}(b, a)$. Покажем замкнутость образа оператора $\mathscr{L}_{\Gamma}$. Рассмотрим последовательность функций $\left(f_{n}\right)$ из $\operatorname{Im} \mathscr{L}_{\Gamma}$, сходящуюся к функции $f_{0}$. Последовательность $\left(x_{n}\right)$, где $x_{n}=\mathscr{C} f_{n}, n=1,2, \ldots$, в силу леммы 3 , принадлежит подпространству $\operatorname{Im}(\Gamma-\mathscr{U}(b, a))$. Заметим, что $\left(x_{n}\right)$ сходится к элементу $x_{0}=\mathscr{C} f_{0}$. В силу замкнутости подпространства $\operatorname{Im}(\Gamma-\mathscr{U}(b, a))$ элемент $x_{0} \in \operatorname{Im}\left(\Gamma-\mathscr{U}(b, a)\right.$, а значит, $f_{0} \in \operatorname{Im} \mathscr{L}_{\Gamma}$ по лемме 3. Теорема доказана.

Теорема 8. Если $\operatorname{Im} \mathscr{L}_{\Gamma}$ - замкнутое дополняемое подпространство в $\mathscr{F}$ и $\mathscr{F}_{0}-$ некоторое замкнутое подпространство из $\mathscr{F}$, для которого

$$
\mathscr{F}=\operatorname{Im} \mathscr{L}_{\Gamma} \oplus \mathscr{F}_{0},
$$

то пространство Х представимо в виде

$$
X=\operatorname{Im}(\Gamma-\mathscr{U}(b, a)) \oplus \mathscr{C}\left(\mathscr{F}_{0}\right),
$$

причем $\mathscr{C}\left(\mathscr{F}_{0}\right)$ - замкнутое подпространство в $X$.

ДокАзАтЕЛьство. Покажем сначала, что любой вектор $x$ из $X$ представим в виде $x=x_{0}+x_{1}$, где $x_{0}$ из $\mathscr{C}\left(\mathscr{F}_{0}\right), x_{1}$ из $\operatorname{Im}(\Gamma-\mathscr{U}(b, a))$. Из разложения (3.5) следует, что функция $\mathscr{B} x$ представима в виде $\mathscr{B} x=f_{0}+f_{1}$, где $f_{0}$ из $\mathscr{F}_{0}$, а $f_{1}$ из $\operatorname{Im} \mathscr{L}_{\Gamma}$. Тогда по лемме 2 получаем, что $x=\mathscr{C} \mathscr{B} x=\mathscr{C} f_{0}+\mathscr{C} f_{1}$. Так как $\mathscr{C} f_{0} \in \mathscr{C} \mathscr{F}$, $\mathscr{C} f_{1} \in \operatorname{Im}(\Gamma-\mathscr{U}(b, a))$ (по лемме 3$)$, то в качестве $x_{0}, x_{1}$ можно взять $x_{0}=\mathscr{C} f_{0}$, $x_{1}=\mathscr{C} f_{1}$.

Покажем единственность разложения. Пусть вектор $x$ также представим в виде $x=x_{0}^{\prime}+x_{1}^{\prime}$, где $x_{0}^{\prime}$ из $\mathscr{C}\left(\mathscr{F}_{0}\right), x_{1}^{\prime}$ из $\operatorname{Im}(\Gamma-\mathscr{U}(b, a))$. Тогда существует такая функция $f_{0}^{\prime}$ из $\mathscr{F}_{0}$, что $x_{0}^{\prime}=\mathscr{C} f_{0}^{\prime}$. Поскольку $\mathscr{C} f_{0}-\mathscr{C} f_{0}^{\prime}=x_{0}-x_{0}^{\prime}=x_{1}-x_{1}^{\prime}$ и $\left(x_{1}-x_{1}^{\prime}\right) \in \operatorname{Im}(\Gamma-\mathscr{U}(b, a))$, то по лемме 3 получаем, что $\left(f_{0}-f_{0}^{\prime}\right) \in \operatorname{Im} \mathscr{L}_{\Gamma}$. Из разложения (3.5) следует, что $f_{0}=f_{1}^{\prime}$.

Замкнутость пространства $\mathscr{C} \mathscr{F} 0$ следует из критерия Като (см. [7; теорема 7.4.20]). Теорема доказана. 
Теорема 9. Если $\operatorname{Im}(\Gamma-\mathscr{U}(b, a))$ - замкнутое дополняемое подпространство в $X$ и $X_{0}$ - некоторое замкнутое подпространство из $X$, для которого

$$
X=\operatorname{Im}(\Gamma-\mathscr{U}(b, a)) \oplus X_{0},
$$

то $\mathscr{F}$ представимо в виде

$$
\mathscr{F}=\operatorname{Im} \mathscr{L}_{\Gamma} \oplus \mathscr{B}\left(X_{0}\right),
$$

где $\mathscr{B}\left(X_{0}\right)$ является замкнутым подпространством из $\mathscr{F}$.

Доказательство. Докажем сначала, что любая функция $f$ из $\mathscr{F}$ представима в виде $f=f_{0}+f_{1}$, где $f_{0} \in \mathscr{B}\left(X_{0}\right), f_{1} \in \operatorname{Im} \mathscr{L}_{\Gamma}$. Из разложения (3.7) следует, что $\mathscr{C} f=x_{0}+x_{1}$, где $x_{0} \in X_{0}, x_{1} \in \operatorname{Im}(\Gamma-\mathscr{U}(b, a))$. В качестве функции $f_{0}$ возьмем функцию $f_{0}=\mathscr{B} x_{0}$. Так как для функции $f_{1}=f-f_{0}$ справедливо

$$
\mathscr{C} f_{1}=\mathscr{C} f-\mathscr{C} f_{0}=x_{1} \in \operatorname{Im}(\Gamma-\mathscr{U}(b, a)),
$$

то по лемме 3 получаем, что $f_{1} \in \operatorname{Im} \mathscr{L}_{\Gamma}$.

Покажем, что полученное представление единственно. Пусть $f=f_{0}^{\prime}+f_{1}^{\prime}$, где $f_{0}^{\prime}=\mathscr{B} x_{0}^{\prime}, x_{0}^{\prime} \in X_{0}, f_{1}^{\prime} \in \operatorname{Im} \mathscr{L}_{\Gamma}$. Так как

$$
x_{0}-x_{0}^{\prime}=\mathscr{C}\left(f_{0}-f_{0}^{\prime}\right)=\mathscr{C}\left(f_{1}-f_{1}^{\prime}\right) \in \operatorname{Im}(\Gamma-\mathscr{U}(b, a)),
$$

то из разложения (3.7) следует, что $x_{0}=x_{0}^{\prime}$, а значит, $f_{0}=f_{0}^{\prime}$.

Замкнутость подпространства $\mathscr{B}\left(X_{0}\right)$ следует из критерия Като (см. [7; теорема 7.4.20]). Теорема доказана.

Введем в рассмотрение линейный оператор $B: X \rightarrow \mathscr{F}$, определяемый равенствами

$$
\left(B x_{0}\right)(t)=\mathscr{U}(t, a) x_{0}, \quad t \in[a, b], \quad x_{0} \in \operatorname{Ker}(\Gamma-\mathscr{U}(b, a)) .
$$

Из доказательства леммы 1 следует, что оператор $B$ ограничен.

Лемма 5. Ядра оператора $\mathscr{L}_{\Gamma}$ и отношения $Г-\mathscr{U}(b, a)$ изоморфны, а их изоморфизм осуществляет оператор $B$.

ДокАЗАтЕЛЬство. Справедливость утверждения непосредственно следует из леммы 3 .

Теорема 10. Если Ker $\mathscr{L}_{\Gamma}$ - замкнутое дополняемое подпространство в пространстве $\mathscr{F} u$

$$
\mathscr{F}=\operatorname{Ker} \mathscr{L}_{\Gamma} \oplus \mathscr{F}_{1},
$$

где $\mathscr{F}_{1}$ - замкнутое подпространство, то $\operatorname{Ker}(\Gamma-\mathscr{U}(b, a))$ - замкнутое дополняемое подпространство из $X$ и

$$
X=\operatorname{Ker}(\Gamma-\mathscr{U}(b, a)) \oplus B^{-1}\left(\mathscr{F}_{1}\right) .
$$

ДоКАЗАтЕЛЬСтво. Отметим, что $B^{-1}\left(\mathscr{F}_{1}\right)$ - замкнутое подпространство как прообраз замкнутого подпространства $\mathscr{F}_{1}$ при непрерывном отображении $B$.

Пусть $x_{0} \in \operatorname{Ker}(\Gamma-\mathscr{U}(b, a)) \cap B^{-1}\left(\mathscr{F}_{1}\right)$. Поскольку $B x_{0} \in \operatorname{Ker} \mathscr{L}_{\Gamma}$ и $B x_{0}=0$, а значит, в силу инъективности оператора $B$ имеем $x_{0}=0$.

Ввиду того, что $B$ осуществляет изоморфизм подпространств Ker $\mathscr{L}_{\Gamma}$ (см. лемму 5), то теперь из (3.10) следует разложение (3.11). Теорема доказана. 
ТеОРема 11. Если замкнутым является оператор $\mathscr{L}_{\Gamma}$, то также замкнутым будет отношение $\Gamma-\mathscr{U}(b, a)$.

ДокАЗАтЕЛЬСтво. Рассмотрим последовательность $\left(z_{n}, y_{n}\right)$ из $(\Gamma-\mathscr{U}(b, a))$, сходящуюся к элементу $\left(z_{0}, y_{0}\right)$. Введем последовательность функций $\left(f_{n}\right)$, определенных по правилу $f_{n}=\mathscr{B} y_{n}, 1 \leqslant n<\infty$. По определению оператора $\mathscr{L}_{\Gamma}$ получим, что существует такая последовательность функций $\left(x_{n}\right)$, для которых $x_{n} \in\left(\mathscr{L}_{\Gamma}^{-1} f_{n}\right)$ и выполняется равенство $\left(x_{n}(a), x_{n}(b)\right)=\left(z_{n}, y_{n}+\mathscr{U}(b, a) z_{n}\right)$ для любого натурального $n$. Отметим, что последовательность $\left(x_{n}, f_{n}\right)$ сходится к элементу $\left(x_{0}, f_{0}\right)$, где $f_{0}=\mathscr{B} y_{0}, x_{0} \in\left(\mathscr{L}_{\Gamma}^{-1} f_{0}\right)$, причем $\left(x_{0}(a), x_{0}(b)\right)=\left(z_{0}, y_{0}+\mathscr{U}(b, a) z_{0}\right)$. В силу замкнутости оператора $\mathscr{L}_{\Gamma}$ получим, что $x_{0} \in D\left(\mathscr{L}_{\Gamma}\right)$, а значит, $\left(z_{0}, y_{0}+\mathscr{U}(b, a) z_{0}\right)=$ $\left(x_{0}(a), x_{0}(b)\right) \in \Gamma$. Следовательно, $\left(z_{0}, y_{0}\right) \in(\Gamma-\mathscr{U}(b, a))$. Теорема доказана.

Оператор $\mathscr{L}_{\Gamma}$ не всегда замкнут для замкнутого отношения $Г$.

ПримеР 1. Пусть оператор $A=A^{*}<0$ - отрицательно определенный самосопряженный оператор в гильбертовом пространстве $H$, имеющий компактную резольвенту. Рассмотрим ортонормированный базис $e_{1}, e_{2}, \ldots$ в $H$, составленный из собственных векторов оператора $A$, т.е. $A e_{k}=\lambda_{k} e_{k}, k \geqslant 1$, предполагая, что $\lambda_{k} \leqslant-k^{3}$, $k \geqslant 1$. Тогда $A$ является генератором сильно непрерывной полугруппы операторов $U: \mathbb{R}_{+} \rightarrow$ End $H$, для которой

$$
U(t) e_{k}=e^{\lambda_{k} t} e_{k}, \quad k \geqslant 0, \quad t \in \mathbb{R} .
$$

Пусть

$$
x_{0}=\sum_{k \geqslant 1} \frac{1}{k} e_{k}
$$

и $E=\left\{x \in H:\left(x, x_{0}\right)=0\right\}-$ подпространство векторов из $H$, перпендикулярных вектору $x_{0}$. В качестве семейства эволюционных операторов возьмем семейство

$$
\mathscr{U}(t, s)=U(t-s), \quad 0 \leqslant s \leqslant t \leqslant 1 .
$$

Последовательность векторов из $H$ вида

$$
x_{n}=\sum_{k=1, k \neq n}^{\infty} \frac{1}{k} e_{k}-\left(n \sum_{k=1, k \neq n}^{\infty} \frac{1}{k^{2}}\right) e_{n}
$$

принадлежит подпространству $E$. Все функции вида

$$
\varphi_{n}(t)=U(t) x_{n}, \quad n \geqslant 1, \quad t \geqslant 0,
$$

содержатся в $\operatorname{Ker} \mathscr{L}_{\Gamma}$ (см. лемму 3), так как $\operatorname{Ker}(\Gamma-\mathscr{U}(b, a))=E$, где $\Gamma=E \times X$, при любом выборе пространства $\mathscr{F}([0,1], H)$, в котором действует оператор $\mathscr{L}_{\Gamma}$, построенный по эволюционному семейству $\mathscr{U}$ и отношению Г. Пусть $\mathscr{F}([0,1], H)=$ $L_{2}([0,1], H)$. Тогда для функции $\varphi_{0}(t)=U(t) x_{0}, t \geqslant 0$, получаем

$$
\begin{aligned}
\left\|\varphi_{n}-\varphi_{0}\right\|^{2} & =\int_{0}^{1}\left\|U(t) x_{n}-U(t) x_{0}\right\|^{2} d t \\
& =\left(\frac{1}{n}+n \sum_{k \geqslant 1, k \neq n} \frac{1}{k^{2}}\right)^{2} \int_{0}^{1} e^{2 \lambda_{n} t} d t \rightarrow 0, \quad n \rightarrow \infty .
\end{aligned}
$$

Поскольку $\varphi_{0}(0)=x_{0} \bar{\in} E$, то $\varphi_{0} \bar{\in} \operatorname{Ker} \mathscr{L}_{\Gamma}$. Итак Ker $\mathscr{L}_{\Gamma}-$ незамкнутое подпротранство в $L_{2}([0,1], H)$ и поэтому $\mathscr{L}_{\Gamma}-$ незамкнутый оператор. 
Tеорема 12. Оператор $\mathscr{L}_{\Gamma}$ непрерывно обратим тогда и только тогда, когда непрерывно обратимо отношение $\Gamma-\mathscr{U}(b, a)$, и обратный оператор представим в виде

$$
\left(\mathscr{L}_{\Gamma}^{-1} f\right)(t)=\int_{a}^{b} G(t, s) f(s) d s \quad \text { для всех } t \in[a, b]
$$

для любой функиии $f \in \mathscr{F}$, где

$$
G(t, s)= \begin{cases}\mathscr{U}(t, a)(\Gamma-\mathscr{U}(b, a))^{-1} \mathscr{U}(b, s)+\mathscr{U}(t, s), & s \leqslant t, \\ \mathscr{U}(t, a)(\Gamma-\mathscr{U}(b, a))^{-1} \mathscr{U}(b, s), & s>t .\end{cases}
$$

ДокАЗАТЕЛЬСтво. Пусть оператор $\mathscr{L}_{\Gamma}$ непрерывно обратим. Тогда из леммы 5 и теоремы 8 следует, что отношение $(\Gamma-\mathscr{U}(b, a))^{-1}$ является линейным оператором, определенным на всем пространстве $X$. Из теоремы 11 следует замкнутость оператора $(\Gamma-\mathscr{U}(b, a))^{-1}$, а значит, и его ограниченность.

Пусть непрерывно обратимо отношение $\Gamma-\mathscr{U}(b, a)$. Обратимость оператора $\mathscr{L}_{\Gamma}$ следует из леммы 5 и теоремы 9.

Покажем теперь, что оператор $K: \mathscr{F} \rightarrow \mathscr{F}$, определенный по правилу

$$
(K f)(t)=\int_{a}^{b} G(t, s) f(s) d s \quad \text { для всех } t \in[a, b],
$$

является обратным к оператору $\mathscr{L}_{\Gamma}$. Возьмем произвольную функцию $f$ из $\mathscr{F}$. Для доказательства утверждения достаточно показать, что $\mathscr{L}_{\Gamma} x=f$, где $x=K f$.

По определению оператора $K$ получаем, что

$$
x(t)=(K f)(t)=\mathscr{U}(t, a) x(a)+\int_{a}^{t} \mathscr{U}(t, s) f(s) d s, \quad t \in[a, b],
$$

где

$$
x(a)=(\Gamma-\mathscr{U}(b, a))^{-1} \int_{a}^{b} \mathscr{U}(b, s) f(s) d s=(\Gamma-\mathscr{U}(b, a))^{-1} \mathscr{C} f .
$$

Таким образом, пара функций $(x, f)$ удовлетворяет равенствам (1.1). Осталось показать, что $(x(a), x(b)) \in \Gamma$. Из равенств $(1.1)$ следует, что $x(b)=U(b, a) x(a)+\mathscr{C} f$. Так как $x(a)=(\Gamma-\mathscr{U}(b, a))^{-1} \mathscr{C} f$, то

$$
x(b)-\mathscr{U}(b, a) x(a)=\mathscr{C} f \in(\Gamma-\mathscr{U}(b, a)) x(a) .
$$

Следовательно, $x(b) \in \Gamma x(a)$, т.е. $(x(a), x(b)) \in \Gamma$. Таким образом, мы показали, что $\mathscr{L}_{\Gamma} x=f$. Ограниченность оператора $\mathscr{L}_{\Gamma}^{-1}$ следует из того факта, что существует такое число $M$, для которого выполняется неравенство $\|\mathscr{U}(t, a)\| \leqslant M$ для любой пары $(t, s)$ из $\Delta$ (см. доказательство леммы 1$)$ и ограниченности оператора $(\Gamma-\mathscr{U}(b, a))^{-1}$. Теорема доказана.

ЗАмечАнИЕ 1 . Отметим, что формула для обратного оператора $\mathscr{L}_{\Gamma}^{-1}$ корректно определена даже в случае, когда $\mathscr{U}$ является семейством эволюционных операторов "вперед", т.е. $\mathscr{U}$ определен только на множестве $\Delta_{1}=\{(t, s) \in \Delta, s \leqslant t\}$. В этом случае непрерывная обратимость отношения $\Gamma-\mathscr{U}(b, a)$ является достаточным условием для непрерывной обратимости оператора $\mathscr{L}_{\Gamma}$. 
ПримеР 2. Покажем, что существуют такое семейство эволюционных операторов "вперед" $\mathscr{U}$ и такое отношение $\Gamma$, что оператор $\mathscr{L}_{\Gamma}$ будет непрерывно обратим, а отношение $\Gamma-\mathscr{U}(b, a)$ не будет непрерывно обратимым. В качестве пространства $X$ рассмотрим пространство непрерывных ограниченных функций $C_{\mathrm{b}}(\mathbb{R}, \mathbb{R})$, а в качестве пространства $\mathscr{F}$ возьмем пространство $C([0,1], X)$. Рассмотрим оператор $A: D(A) \subset X \rightarrow X, A x=\ddot{x}, x \in D(A)$, с областью определения $D(A)$, состоящей из всех функций $x \in X$, таких, что $\dot{x}$ и $\ddot{x}$ принадлежат $X$. В [6] было показано, что оператор $A$ является генератором полугруппы $T: \mathbb{R}_{+} \rightarrow \operatorname{End} X$, определенной по формуле

$$
(T(t) x)(s)=\frac{1}{2 \sqrt{\pi t}} \int_{-\infty}^{+\infty} e^{-\tau^{2} / 4 t} x(\tau+s) d \tau, \quad t>0 .
$$

Семейство эволюционных операторов $\mathscr{U}: \Delta_{1} \rightarrow X$, где $\Delta_{1}=\{(t, s): t, s \in[0,1], s \leqslant t\}$, определим по правилу $\mathscr{U}(t, s)=T(t-s)$ для любой пары $(t, s)$ из $\Delta_{1}$. Рассмотрим оператор $P: D(P) \subset X \rightarrow X, P x=\dot{x}, x \in D(P)$ с областью определения $D(P)$, состоящей из всех функций $x \in X$ таких, что $\dot{x}$ принадлежит $X$. Отношение $\Gamma$ возьмем равным оператору $T(1)+I_{D(P)}$, где $I_{D(P)}$ - тождественный оператор на $D(P)$. Тогда $\Gamma-\mathscr{U}(b, a)=I_{D(P)}$. В [6; лемма VIII.1.21] было доказано, что $\mathscr{C} f \in D(P)$. Так как лемма 3 справедлива и в случае, когда $\mathscr{U}$ - семейство эволюционных операторов "вперед", то, так как $\Gamma-\mathscr{U}(b, a)=I_{D(P)}$, оператор $\mathscr{L}_{\Gamma}$ обратим. Оператор, обратный к оператору $\mathscr{L}_{\Gamma}$, имеет вид

$$
\begin{aligned}
\left(\mathscr{L}_{\Gamma}^{-1} f\right)(t) & =\mathscr{U}(t, 0)(\Gamma-\mathscr{U}(1,0))^{-1} \mathscr{C} f+\int_{0}^{t} \mathscr{U}(t, s) f(s) d s \\
& =T(t) \mathscr{C} f+\int_{0}^{t} T(t-s) f(s) d s \quad \text { для всех } f \in \mathscr{F} .
\end{aligned}
$$

Доказательство леммы 1 справедливо и для случая, когда $\mathscr{U}$ - семейство эволюционных операторов "вперед”. Поэтому оператор $\mathscr{C}$ ограничен и существует константа $M$ такая, что $\|T(t-s)\| \leqslant M$ для любой пары $(t, s)$ из $\Delta_{1}$. Отсюда следует ограниченность оператора $\mathscr{L}_{\Gamma}^{-1}$. Таким образом, оператор $\mathscr{L}_{\Gamma}$ непрерывно обратим, тогда как $\Gamma-\mathscr{U}(1,0)=I_{D(P)}$ не является непрерывно обратимым отношением.

Из леммы 5 и теорем 8, 9 вытекает следующая

Теорема 13. Оператор $\mathscr{L}_{\Gamma}$ фредгольмов тогда и толъко тогда, когда фредгольмовым является отношение Г- И $(b, a)$. Если один из них фредгольмов, то

$\operatorname{dim} \operatorname{Ker} \mathscr{L}_{\Gamma}=\operatorname{dim} \operatorname{Ker}(\Gamma-\mathscr{U}(b, a)), \quad \operatorname{Codim} \operatorname{Im} \mathscr{L}_{\Gamma}=\operatorname{Codim} \operatorname{Im}(\Gamma-\mathscr{U}(b, a))$, а значит, и их индексы совпадают.

ЗАмечАнИЕ 2. Из равенств (1.1), определяющих оператор $\mathscr{L}_{\Gamma}$, следует, что для любого $\lambda \in \mathbb{K}$ оператор $\mathscr{L}_{\Gamma}-\lambda I$ задается (с помощью тех же равенств) по семейству эволюционных операторов $\mathscr{U}_{\lambda}: \Delta \rightarrow$ End $X$ вида

$$
\mathscr{U}_{\lambda}(t, s)=e^{\lambda(t-s)} \mathscr{U}(t, s), \quad(t, s) \in \Delta .
$$

Обозначим символом $\sigma(\Gamma, \mathscr{U}(b, a))$ множество вида

$$
\sigma(\Gamma, \mathscr{U}(b, a))=\left\{\lambda \in \mathbb{K}:(\Gamma-\lambda \mathscr{U}(b, a))^{-1} \bar{\epsilon} \text { End } X\right\} .
$$


Из замечания 2 и теоремы 12 вытекают следующие две теоремы.

TeOpema 14. Cnектр оператора $\mathscr{L}_{\Gamma}$ uмеeт вид

$$
\sigma\left(\mathscr{L}_{\Gamma}\right)=\left\{\lambda \in \mathbb{K}: e^{\lambda(b-a)} \in \sigma(\Gamma, \mathscr{U}(b, a))\right\}
$$

СледСТВИЕ 1. Пусть $X$ - комплексное банахово пространство. Тогда если спектр оператора $\mathscr{L}_{\Gamma}$ не пуст, то для него справедливо следующее равенство:

$$
\bigcup_{k \in \mathbb{Z}}\left(\sigma\left(\mathscr{L}_{\Gamma}\right)+\frac{i 2 \pi k}{b-a}\right)=\sigma\left(\mathscr{L}_{\Gamma}\right)
$$

Tеорема 15. Резольвента оператора $\mathscr{L}_{\Gamma} R\left(\cdot, \mathscr{L}_{\Gamma}\right): \rho\left(\mathscr{L}_{\Gamma}\right) \rightarrow$ End $\mathscr{F}$ имеет вид

$$
\left(R\left(\lambda, \mathscr{L}_{\Gamma}\right) f\right)(s)=\int_{a}^{b} G(\lambda, t, s) f(s) d s
$$

для любой функции $f$ из $\mathscr{F}$, где

$$
G(\lambda, t, s)= \begin{cases}e^{\lambda(t-s)}\left[\mathscr{U}(t, a)\left(e^{\lambda(a-b)} \Gamma-\mathscr{U}(b, a)\right)^{-1} \mathscr{U}(b, s)+\mathscr{U}(t, s)\right], & s \leqslant t, \\ e^{\lambda(t-s)} \mathscr{U}(t, a)\left(e^{\lambda(a-b)} \Gamma-\mathscr{U}(b, a)\right)^{-1} \mathscr{U}(b, s), & s>t .\end{cases}
$$

Введем следующие обозначения. Символом $\sigma_{\mathrm{d}}(\Gamma, \mathscr{U}(b, a))$ обозначим дискретный спектр пары $(\Gamma, \mathscr{U}(b, a))$, т.е. множество тех $\lambda \in \sigma(\Gamma, \mathscr{U}(b, a))$, для которых отношение $\Gamma-\lambda \mathscr{U}(b, a)$ не инъективно. Символом $\sigma_{\mathrm{c}}(\Gamma, \mathscr{U}(b, a))$ обозначим непрерывный спектр пары $(\Gamma, \mathscr{U}(b, a))$, т.е. множество тех $\lambda \in \sigma(\Gamma, \mathscr{U}(b, a))$, для которых отношение $\Gamma-\lambda \mathscr{U}(b, a)$ инъективно и замыкание пространства $\operatorname{Im}(\Gamma-\mathscr{U}(b, a))$ совпадает с $X$. Символом $\sigma_{\mathrm{r}}(\Gamma, \mathscr{U}(b, a))$ обозначим остаточный спектр пары $(\Gamma, \mathscr{U}(b, a))$, т.е. множество тех $\lambda \in \sigma(\Gamma, \mathscr{U}(b, a))$, для которых отношение $\Gamma-\lambda \mathscr{U}(b, a)$ инъективно и замыкание пространства $\operatorname{Im}(\Gamma-\mathscr{U}(b, a))$ не совпадает с $X$. Очевидно, что множества $\sigma_{\mathrm{d}}(\Gamma, \mathscr{U}(b, a)), \sigma_{\mathrm{c}}(\Gamma, \mathscr{U}(b, a)), \sigma_{\mathrm{r}}(\Gamma, \mathscr{U}(b, a))$ попарно не пересекаются и

$$
\sigma(\Gamma, \mathscr{U}(b, a))=\sigma_{\mathrm{d}}(\Gamma, \mathscr{U}(b, a)) \cup \sigma_{\mathrm{c}}(\Gamma, \mathscr{U}(b, a)) \cup \sigma_{\mathrm{r}}(\Gamma, \mathscr{U}(b, a)) .
$$

Теорема 16. Для оператора $\mathscr{L}_{\Gamma}$ справедливъ следующие равенства:

$$
\begin{aligned}
\sigma_{\mathrm{d}}\left(\mathscr{L}_{\Gamma}\right) & =\left\{\lambda: e^{\lambda(b-a)} \in \sigma_{\mathrm{d}}(\Gamma, \mathscr{U}(b, a))\right\}, \\
\sigma_{\mathrm{c}}\left(\mathscr{L}_{\Gamma}\right) & =\left\{\lambda: e^{\lambda(b-a)} \in \sigma_{\mathrm{c}}(\Gamma, \mathscr{U}(b, a))\right\}, \\
\sigma_{\mathrm{r}}\left(\mathscr{L}_{\Gamma}\right) & =\left\{\lambda: e^{\lambda(b-a)} \in \sigma_{\mathrm{r}}(\Gamma, \mathscr{U}(b, a))\right\} .
\end{aligned}
$$

ДокАзАтельство. Докажем равенство (3.18). Из леммы 3 и замечания 2 следует, что

$$
\operatorname{Ker}\left(\mathscr{L}_{\Gamma}-\lambda I\right)=\left\{x \in \mathscr{F}: x=e^{\lambda(t-a)} x_{0}, x_{0} \in \operatorname{Ker}\left(\Gamma-e^{\lambda(b-a)} \mathscr{U}(b, a)\right)\right\} .
$$

Отсюда непосредственно следует равенство (3.18).

Докажем равенство (3.19). Пусть $\lambda \in \sigma_{\mathrm{c}}\left(\mathscr{L}_{\Gamma}\right)$. Покажем, что $e^{\lambda(b-a)}$ принадлежит множеству $\sigma_{\mathrm{c}}(\Gamma, \mathscr{U}(b, a))$. По лемме 3 получаем, что $\operatorname{Ker}\left(\Gamma-e^{\lambda(b-a)} \mathscr{U}(b, a)\right)=\{0\}$. Покажем, что замыкание пространства $\operatorname{Im}\left(\Gamma-e^{\lambda(b-a)} \mathscr{U}(b, a)\right)$ совпадает с $X$. Возьмем произвольный элемент $x$ из $X$. Так как пространство $\operatorname{Im}\left(\mathscr{L}_{\Gamma}-\lambda I\right)$ плотно в $\mathscr{F}$, 
то существует последовательность функций $\left(f_{n}\right)$ из $\operatorname{Im}\left(\mathscr{L}_{\Gamma}-\lambda I\right)$, сходящаяся к функции $\mathscr{B} x$. Поскольку оператор $\mathscr{B}$ ограничен (по лемме 1$)$, последовательность $\left(x_{n}\right)$, где $x_{n}=\mathscr{C} f_{n}, n \geqslant 1$, сходится к элементу $\mathscr{C} f=\mathscr{C} \mathscr{B} x=x$. По лемме 3 получаем, что $x_{n} \in \operatorname{Im}\left(\Gamma-e^{\lambda(b-a)} \mathscr{U}(b, a)\right)$ для любого натурального числа $n$. Следовательно, замыкание пространства $\operatorname{Im}\left(\Gamma-e^{\lambda(b-a)} \mathscr{U}(b, a)\right)$ совпадает с $X$.

Пусть теперь $e^{\lambda(b-a)} \in \sigma_{\mathrm{c}}(\Gamma, \mathscr{U}(b, a))$. Покажем, что $\lambda \in \sigma_{\mathrm{c}}\left(\mathscr{L}_{\Gamma}\right)$. Из леммы 3 следует, что оператор $\mathscr{L}_{\Gamma}-\lambda I$ является инъективным. Покажем, что замыкание пространства $\operatorname{Im}\left(\mathscr{L}_{\Gamma}-\lambda I\right)$ есть $\mathscr{F}$. Возьмем произвольную функции $f$ из $\mathscr{F}$. Пусть $x=C f$. Тогда функция $f$ представима в виде $f=g+B x$, где $g \in \operatorname{Ker} \mathscr{C}$. Из леммы 3 следует, что $g \in \operatorname{Im}\left(\mathscr{L}_{\Gamma}-\lambda I\right)$. Так как пространство $\operatorname{Im}\left(\Gamma-e^{\lambda(b-a)} \mathscr{U}(b, a)\right)$ плотно в $X$, то существует последовательность $\left(x_{n}\right)$ из $\operatorname{Im}\left(\Gamma-e^{\lambda(b-a)} \mathscr{U}(b, a)\right)$, сходящаяся к элементу $x$. Поскольку $\mathscr{B} x_{n} \in \operatorname{Im}\left(\mathscr{L}_{\Gamma}-\lambda I\right)$ для любого натурального $n$ (см. лемму 3 , то $f_{n}=\left(g+\mathscr{B} x_{n}\right) \in \operatorname{Im}\left(\mathscr{L}_{\Gamma}-\lambda I\right)$. Последовательность функций $\left(f_{n}\right)$ сходится к функции $f$. Следовательно, пространство $\operatorname{Im}\left(\mathscr{L}_{\Gamma}-\lambda I\right)$ плотно в $\mathscr{F}$. Равенство (3.19) доказано.

Равенство (3.20) следует из теоремы 14 и того факта, что множества $\sigma_{\mathrm{d}}(\Gamma, \mathscr{U}(b, a))$, $\sigma_{\mathrm{c}}(\Gamma, \mathscr{U}(b, a)), \sigma_{\mathrm{r}}(\Gamma, \mathscr{U}(b, a))$ попарно не пересекаются и

$$
\sigma(\Gamma, \mathscr{U}(b, a))=\sigma_{\mathrm{d}}(\Gamma, \mathscr{U}(b, a)) \cup \sigma_{\mathrm{c}}(\Gamma, \mathscr{U}(b, a)) \cup \sigma_{\mathrm{r}}(\Gamma, \mathscr{U}(b, a)) .
$$

Лемма 6. Для оператора $\mathscr{L}_{\Gamma}$ имеет место следующее свойство:

$$
\gamma\left(\mathscr{L}_{\Gamma}\right)>0 \quad \Longleftrightarrow \quad \gamma(\Gamma-\mathscr{U}(b, a))>0
$$

ДокАЗАТЕЛьство. Как следует из теоремы 3, для доказательства утверждения достаточно показать, что отношение $\mathscr{L}_{\Gamma}^{-1}$ ограничено тогда и только тогда, когда ограниченным является отношение $(\Gamma-\mathscr{U}(b, a))^{-1}$.

Пусть ограниченным является отношение $(\Gamma-\mathscr{U}(b, a))^{-1}$. Покажем, что ограниченным является отношение $\mathscr{L}_{\Gamma}^{-1}$. Из определения оператора $\mathscr{L}_{\Gamma}$ следует, что для любой функции $x$ из $\mathscr{L}_{\Gamma}^{-1} f$, где $f \in \operatorname{Im} \mathscr{L}_{\Gamma}$, справедливо равенство

$$
x(t)=\mathscr{U}(t, a) x_{0}+\int_{a}^{t} \mathscr{U}(t, s) f(s) d s, \quad t \in[a, b],
$$

где $x_{0}-$ некоторый элемент из $(\Gamma-\mathscr{U}(b, a))^{-1} \mathscr{C} f$. Из последнего равенства в силу ограниченности отношения $(\Gamma-\mathscr{U}(b, a))^{-1}$, ограниченности оператора $\mathscr{C}$ и оценки $\|\mathscr{U}(t, s)\| \leqslant M$ (см. доказательство леммы 1) следует ограниченность отношения $\mathscr{L}_{\Gamma}^{-1}$.

Пусть отношение $(\Gamma-\mathscr{U}(b, a))^{-1}$ не ограничено. Покажем, что неограниченным является отношение $\mathscr{L}_{\Gamma}^{-1}$. Отметим, что так как $\|\mathscr{U}(t, s)\| \leqslant M$ для любой пары $(t, s)$ из $\Delta$, то в силу обратимости оператора $\mathscr{U}(t, s)$ при любых $(t, s) \in \Delta$, получим оценку $\|\mathscr{U}(t, s)\| \geqslant 1 / M$. Из этой оценки и равенства (3.22) следует, что для любой функции $x$ из $\mathscr{L}_{\Gamma}^{-1} f$, где $f \in \operatorname{Im} \mathscr{L}_{\Gamma},\|f\|<1$, выполняется неравенство

$$
\|x\| \geqslant \frac{1}{M}\|x(a)\|-M_{1}
$$

где $M_{1}=M(b-a)^{1 / p}(b-a+1)$ для $\mathscr{F}=L_{p}[a, b], 1 \leqslant p<\infty$, и $M_{1}=M(b-a)$, для $\mathscr{F} \in\left\{L_{\infty}[a, b], C[a, b]\right\}$. Так как $x(a) \in(\Gamma-\mathscr{U}(b, a))^{-1} \mathscr{C} f$, то из последнего неравенства в силу неограниченности отношения $(\Gamma-\mathscr{U}(b, a))^{-1}$ следует неограниченность отношения $\mathscr{L}_{\Gamma}^{-1}$. Теорема доказана. 
Из полученных в этом пункте лемм и теорем следует

Теорема 17. Если для оператора $\mathscr{L}_{\Gamma}$ выполнено одно из приведенных в определении 1 десяти свойств, то соответствующим свойством обладает отношение $\Gamma-\mathscr{U}(b, a)$. Любое из десяти свойств определения 1 , выполненное для отношения $\Gamma-\mathscr{U}(b, a)$, исключая, быть может, первое и пятое свойства, выполнено и для onepamopa $\mathscr{L}_{\Gamma}$.

4. Случай упорядоченной пары линейных операторов. Пусть $(A, B)$ упорядоченная пара линейных операторов, где $A, B$ из End $X$. Рассмотрим краевые условия, заданные следующими линейными отношениями:

$$
\begin{aligned}
& \left.\Gamma_{1}=\{(A x, B x), x \in X\}\right)=B A^{-1}, \\
& \Gamma_{2}=\{(x, y) \in X \times X: A x=B y\}=B^{-1} A .
\end{aligned}
$$

ТЕОРема 18. Если для упорядоченной пары $(B, \mathscr{U}(b, a) A)$ резолъвентное множество непусто, то для оператора $\mathscr{L}_{\Gamma_{1}}$ выполняется равенство

$$
\sigma\left(\mathscr{L}_{\Gamma_{1}}\right)=\left\{\lambda \in \mathbb{K}: e^{\lambda(b-a)} \in \sigma(B, \mathscr{U}(b, a) A)\right\} .
$$

ДокАЗАТЕЛЬСтво. Из теоремы 14 следует, что нам достаточно показать, что отношение $\Gamma_{1}-\lambda \mathscr{U}(b, a)$ непрерывно обратимо тогда и только тогда, когда непрерывно обратимым является оператор $B-\lambda \mathscr{U}(b, a) A$. Имеет место следующая цепочка равенств:

$$
\left(B A^{-1}-\lambda \mathscr{U}(b, a)\right)^{-1}=\left((B-\lambda \mathscr{U}(b, a) A) A^{-1}\right)^{-1}=A(B-\lambda \mathscr{U}(b, a) A)^{-1} .
$$

В силу непрерывной обратимости $\mathscr{U}(b, a)$ отношение $A(B-\lambda \mathscr{U}(b, a) A)^{-1}$ принадлежит End $X$ тогда и только тогда, когда

$$
\mathscr{U}(b, a) A(B-\lambda \mathscr{U}(b, a) A)^{-1} \in \operatorname{End} X .
$$

Из теоремы 4 и равенства (2.4) следует, что принадлежность отношения

$$
\mathscr{U}(b, a) A(B-\lambda \mathscr{U}(b, a) A)^{-1}
$$

к End $X$ равносильна непрерывной обратимости оператора $(B-\lambda \mathscr{U}(b, a) A)$. Таким образом, мы показали, что отношение $\Gamma_{1}-\lambda \mathscr{U}(b, a)$ непрерывно обратимо тогда и только тогда, когда непрерывно обратимым является оператор $B-\lambda \mathscr{U}(b, a) A$. Теорема доказана.

Теорема 19. Если для упорядоченной пары $(A, B \mathscr{U}(b, a))$ резолъвентное множество непусто, то для оператора $\mathscr{L}_{\Gamma_{2}}$ выполняется равенство

$$
\sigma\left(\mathscr{L}_{\Gamma_{2}}\right)=\left\{\lambda \in \mathbb{K}: e^{\lambda(b-a)} \in \sigma(A, B \mathscr{U}(b, a))\right\} .
$$

ДокАЗАТЕЛЬСтво. Из теоремы 14 следует, что нам достаточно показать, что отношение $\Gamma_{2}-\lambda \mathscr{U}(b, a)$ непрерывно обратимо тогда и только тогда, когда непрерывно обратимым является оператор $A-\lambda B \mathscr{U}(b, a)$. Имеет место следующая цепочка равенств:

$$
\left(B^{-1} A-\lambda \mathscr{U}(b, a)\right)^{-1}=\left(B^{-1}(A-\lambda B \mathscr{U}(b, a))\right)^{-1}=(A-\lambda B \mathscr{U}(b, a))^{-1} B .
$$


В силу непрерывной обратимости $\mathscr{U}(b, a)$ отношение $(A-\lambda B \mathscr{U}(b, a))^{-1} B$ принадлежит $\operatorname{End} X$ тогда и только тогда, когда

$$
(A-\lambda B \mathscr{U}(b, a))^{-1} B \mathscr{U}(b, a) \in \operatorname{End} X .
$$

Из теоремы 4 и равенства (2.3) следует, что принадлежность отношения

$$
(A-\lambda B \mathscr{U}(b, a))^{-1} B \mathscr{U}(b, a)
$$

к End $X$ равносильна непрерывной обратимости оператора $(A-\lambda B \mathscr{U}(b, a))$. Таким образом, мы показали, что отношение $\Gamma_{2}-\lambda \mathscr{U}(b, a)$ непрерывно обратимо тогда и только тогда, когда непрерывно обратимым является оператор $A-\lambda B \mathscr{U}(b, a)$. Теорема доказана.

Tеорема 20. Резольвента $R\left(\cdot, \mathscr{L}_{\Gamma_{1}}\right): \rho\left(\mathscr{L}_{\Gamma_{1}}\right) \rightarrow$ End $\mathscr{F}$ оператора $\mathscr{L}_{\Gamma_{1}}$ uмеeт вид

$$
\left(R\left(\lambda, \mathscr{L}_{\Gamma_{1}}\right) f\right)(s)=\int_{a}^{b} G(\lambda, t, s) f(s) d s
$$

для любой функции $f$ из $\mathscr{F}$. Функиия $G(t, s)$ задается формулой

$$
G(\lambda, t, s)= \begin{cases}e^{\lambda(t-s)}\left[\mathscr{U}(t, a) D_{\lambda} \mathscr{U}(b, s)+\mathscr{U}(t, s)\right], & s \leqslant t, \\ e^{\lambda(t-s)} \mathscr{U}(t, a) D_{\lambda} \mathscr{U}(b, s), & s>t,\end{cases}
$$

где $D_{\lambda}=A\left(e^{\lambda(a-b)} B-\mathscr{U}(b, a) A\right)^{-1}$.

ДокАзАтЕЛЬство. Утверждение вытекает из теоремы 15 и равенства

$$
\left(\lambda \Gamma_{1}-\mathscr{U}(b, a)\right)^{-1}=A(\lambda B-\mathscr{U}(b, a) A)^{-1},
$$

которое выполняется для всех $\lambda \neq 0$.

Теорема 21. Резолъвента $R\left(\cdot, \mathscr{L}_{\Gamma_{2}}\right): \rho\left(\mathscr{L}_{\Gamma_{2}}\right) \rightarrow$ End $\mathscr{F}$ оператора $\mathscr{L}_{\Gamma_{2}}$ имеет вид

$$
\left(R\left(\lambda, \mathscr{L}_{\Gamma_{2}}\right) f\right)(s)=\int_{a}^{b} G(\lambda, t, s) f(s) d s
$$

для любой функции $f$ из $\mathscr{F}$. Функция $G(t, s)$ задается формулой

$$
G(\lambda, t, s)= \begin{cases}e^{\lambda(t-s)}\left[\mathscr{U}(t, a) D_{\lambda} \mathscr{U}(b, s)+\mathscr{U}(t, s)\right], & s \leqslant t, \\ e^{\lambda(t-s)} \mathscr{U}(t, a) D_{\lambda} \mathscr{U}(b, s), & s>t,\end{cases}
$$

где $D_{\lambda}=\left(e^{\lambda(a-b)} A-B \mathscr{U}(b, a)\right)^{-1} B$.

ДокАЗАТЕЛЬСтво. Утверждение вытекает из теоремы 15 и равенства

$$
\left(\lambda \Gamma_{2}-\mathscr{U}(b, a)\right)^{-1}=(\lambda A-B \mathscr{U}(b, a))^{-1} B,
$$

которое выполняется для всех $\lambda \neq 0$. 


\section{СПИСОК ЦИТИРОВАННОЙ ЛИТЕРАТУРЫ}

[1] Ф. Аткинсон, Дискретные и непрерывные граничные задачи, Мир, М., 1968.

[2] R. Cross, Multivalued Linear Operators, Monogr. Textbooks Pure Appl. Math., 213, Marcel Dekker, New York, 1998.

[3] А. Г. Баскаков, "Спектральный анализ дифференциальных операторов с неограниченными операторными коэффициентами, разностные отношения и полугруппы разностных отношений”, Изв. РАН. Сер. матем., 73:2 (2009), 3-68.

[4] А. Г. Баскаков, К. И. Чернышов, "Спектральный анализ линейных отношений и вырожденные полугруппы операторов", Матем. сб., 193:11 (2002), 3-42.

[5] В. Б. Диденко, "К спектральной теории упорядоченных пар линейных операторов на конечномерных пространствах", Вестник Воронежск. гос. ун-та. Сер. физ.-матем., 2007, № 2, 104-107.

[6] Н. Данфорд, Дж. Шварц, Линейные операторы. т. 1: Общая теория, ИЛ, М., 1962.

[7] С. С. Кутателадзе, Основы функиионального анализа, Современная математика - студентам и аспирантам, Изд-во Ин-та матем., Новосибирск, 1995.

В. Б. Диденко

Воронежский государственный университет

E-mail:_dor_@mail.ru
Поступило 13.01.2009 